\title{
A New Conformable Fractional Derivative and Applications
}

\author{
Ahmed Kajouni, Ahmed Chafiki $\mathbb{D}$, Khalid Hilal, and Mohamed Oukessou
}

Laboratory of Applied Mathematics and Scientific Competing, Faculty of Sciences and Technics, Sultan Moulay Slimane University, Beni Mellal, Morocco

Correspondence should be addressed to Ahmed Chafiki; ahmedchafiki815@gmail.com

Received 9 September 2021; Accepted 3 November 2021; Published 26 November 2021

Academic Editor: Gaston Mandata N gu r kata

Copyright (c) 2021 Ahmed Kajouni et al. This is an open access article distributed under the Creative Commons Attribution License, which permits unrestricted use, distribution, and reproduction in any medium, provided the original work is properly cited.

\begin{abstract}
This paper is motivated by some papers treating the fractional derivatives. We introduce a new definition of fractional derivative which obeys classical properties including linearity, product rule, quotient rule, power rule, chain rule, Rolle's theorem, and the mean value theorem. The definition $\left(D^{\alpha} f\right)(t)=\lim \left(\left(f\left(t+h e^{(\alpha-1) t}\right)-f(t)\right) / h\right)$, for all $t>0$, and $\alpha \in(0,1)$. If $\alpha=0$, this definition coincides to the classical definition of the first order of the function $f$.
\end{abstract}

\section{Introduction}

The objective of fractional calculus is to generalize traditional derivatives to noninteger orders, see [1-4]. As is well known, many dynamic systems are best characterized by a dynamic fractional-order model, generally based on the notion of differentiation or integration of noninteger order. The study of fractional order systems is more delicate than for their whole order counterparts. Indeed, fractional systems are, on the one hand, considered as memory systems, in particular to take into account the initial conditions, and on the other hand, they present a much more complex dynamic system.

The theory of fractional derivative is a very old theory, which dates back to a conversation on September 30, 1695, between Hôpital and Leibniz concerning the definition of the operator $d^{n} / d x^{n}$ for $n=1 / 2$. Thus, as the time progresses, certain approaches have been given in the literature such as the definition of Riemann-Liouville and that of Caputo.

Recently, the authors in [5] and in [6] define new wellbehaved simple fractional derivatives called the conformable fractional derivative depending just on the basic limit definition of the derivative.

Khalil et al. [5] have introduced a new derivative called the conformable fractional derivative of $f$ of order $\alpha$ and is defined by

$$
T_{\alpha} f(t)=\lim _{\varepsilon \longrightarrow 0} \frac{f\left(t+\varepsilon t^{1-\alpha}\right)-f(t)}{\varepsilon},
$$

for a function $f:[0,+\infty) \longrightarrow \mathbb{R}$ and $0<\alpha \leq 1$, and the fractional derivative at 0 is defined as $f^{(\alpha)}(0)=\lim _{t \rightarrow 0^{+}} T_{\alpha}(f)(t)$.

They then defined the fractional derivative of higher order (i.e., of order $\alpha>1$ ). They also defined the fractional integral of order $0<\alpha \leq 1$ only. They then proved the product rule, and the fractional mean value theorem solved some (conformable) fractional differential equations where the fractional exponential function $e^{t^{\alpha} / \alpha}$ played an important rule.

Katugampola introduced in [6] the new derivative which is defined by

$$
D^{\alpha}(f)(t)=\lim _{\varepsilon \longrightarrow 0} \frac{f\left(t e^{\varepsilon t^{-\alpha}}\right)-f(t)}{\varepsilon},
$$

for $t>0, \alpha \in(0,1)$. If $f$ is $\alpha$ differentiable in some $(0, a), a>0$, and $\lim _{t \rightarrow 0^{+}} D^{\alpha}(f)(t)$ exists, then define

$$
D^{\alpha}(f)(0)=\lim _{t \longrightarrow 0^{+}} D^{\alpha}(f)(t) .
$$

As a consequence of the above definitions, the authors in $[5,6]$ showed that the $\alpha$ derivatives obey the product rule and quotient rule and have results similar to Rolle's theorem and the mean value theorem in classical calculus. 
The purpose of this work is to further generalize the results obtained in [5] and in [6] and introduce a new conformable fractional derivative as the most natural extension of the familiar limit definition of the derivative of a function $f$ at a point.

Remark 1. If $f$ and $T_{\alpha} f(t)$ or $D^{\alpha}(f)(t)$ are differentiable, we have

$$
\frac{d}{\mathrm{~d} t} T_{\alpha} f(t)=(1-\alpha) t^{-\alpha} \frac{d}{\mathrm{~d} t} f(t)+t^{1-\alpha} \frac{d^{2}}{\mathrm{~d} t^{2}} f(t),
$$

(for the derivative in [5]) and

$$
\frac{d}{\mathrm{~d} t} D^{\alpha}(f)(t)=(1-\alpha) t^{-\alpha} \frac{d}{\mathrm{~d} t} f(t)+t^{1-\alpha} \frac{d^{2}}{\mathrm{~d} t^{2}} f(t),
$$

(for the derivative in [6]).

These expressions tends to infinity when $t$ is very small, but this brings regularities in several mathematical problems especially when one seeks to bounded $T_{\alpha} f(t)$ as well as these integer derivatives $(d / \mathrm{d} t) T_{\alpha} f(t),\left(d^{2} / \mathrm{d} t^{2}\right) T_{\alpha} f(t) \ldots$ (generalized function theory, for example).

This remark is the main motivation for our definition:

$$
\left(D^{\alpha} f\right)(t)=\lim _{h \longrightarrow 0} \frac{f\left(t+h e^{(\alpha-1) t}\right)-f(t)}{h} .
$$

\section{New Fractional Derivative}

Definition 1. Given a function $f:[0, \infty) \longrightarrow \mathbb{R}$, and then the conformable fractional derivative of $f$ order $\alpha$ is defined by

$$
\left(D^{\alpha} f\right)(t)=\lim _{h \longrightarrow 0} \frac{f\left(t+h e^{(\alpha-1) t}\right)-f(t)}{h},
$$

for all $t>0$, and $\alpha \in(0,1)$.

If $f$ is $\alpha$ differentiable in some $(0, a), a>0$, and $\lim _{t \rightarrow 0^{+}}\left(D^{\alpha} f\right)(t)$ exists, then define

$$
\left(D^{\alpha} f\right)(0)=\lim _{t \longrightarrow 0^{+}}\left(D^{\alpha} f\right)(t) \text {. }
$$

Theorem 1. If a function $f:[0,+\infty) \longrightarrow \mathbb{R}$ and $\alpha$ differentiable at $t_{0}>0$, then $f$ is continuous at $t_{0}$.

Proof. Since $\quad f\left(t_{0}+h e^{(\alpha-1) t_{0}}\right)-f\left(t_{0}\right)=\quad\left(f\left(t_{0}+\right.\right.$ $\left.\left.h e^{(\alpha-1) t_{0}}\right)-f\left(t_{0}\right)\right) / h \times h$, then $\lim _{h \longrightarrow 0}\left[f\left(t_{0}+h e^{(\alpha-1) t_{0}}\right)-\right.$ $\left.f\left(t_{0}\right)\right]=\lim _{h \longrightarrow 0}\left(\left(f\left(t_{0}+h e^{(\alpha-1) t_{0}}\right)-f\left(t_{0}\right)\right) / h\right) \times \lim _{h \longrightarrow 0} h$.
Let $=h e^{(\alpha-1) t_{0}}$. $\lim _{\varepsilon \longrightarrow 0}\left[f\left(t_{0}+\varepsilon\right)-f\left(t_{0}\right)\right]=f^{(\alpha)}\left(t_{0}\right) \times 0$, which implies that $\lim _{\varepsilon \rightarrow 0} f\left(t_{0}+\varepsilon\right)=f\left(t_{0}\right)$.

Hence, $f$ is continuous at $t_{0}$.

Theorem 2. Let $0<\alpha \leq 1$ and $f, g$ be $\alpha$ differentiable at a point $t>0$. Then,

(1) $D^{\alpha}(a f+b g)=a\left(D^{\alpha} f\right)+b\left(D^{\alpha} g\right)$, for all $a, b \in \in \mathbb{R}$.

(2) $D^{\alpha}\left(t^{p}\right)=p e^{(\alpha-1) t} t^{p-1}$ for all $p \in \mathbb{R}$.

(3) $D^{\alpha}(\lambda)=0$, for all constant functions $f(t)=\lambda$.

(4) $\left(D^{\alpha} f g\right)=f\left(D^{\alpha} g\right)+g\left(D^{\alpha} f\right)$.

(5) $\left(D^{\alpha}(f / g)\right)=\left(f\left(D^{\alpha} g\right)+g\left(D^{\alpha} f\right)\right) / g^{2}$.

(6) If in addition, $f$ is differentiable, then $\left(D^{\alpha} f\right)(t)=e^{(\alpha-1) t} f^{\prime}(t)$.

Proof. Parts (1) through (3) follow directly from the definition. We choose to prove (4) and (6) only since they are crucial. Now, for fixed $t>0$,

$$
\begin{aligned}
\left(D^{\alpha} f g\right)(t) & =\lim _{h \rightarrow 0} \frac{f\left(t+h e^{(\alpha-1) t}\right) g\left(t+h e^{(\alpha-1) t}\right)-f(t) g(t)}{h} \\
& =\lim _{h \longrightarrow 0} \frac{f\left(t+h e^{(\alpha-1) t}\right) g\left(t+h e^{(\alpha-1) t}\right)-f(t) g\left(t+h e^{(\alpha-1) t}\right)+f(t) g\left(t+h e^{(\alpha-1) t}\right)-f(t) g(t)}{h} \\
& =\lim _{h \rightarrow 0} \frac{f\left(t+h e^{(\alpha-1) t}\right)-f(t)}{h} g\left(t+h e^{(\alpha-1) t}\right)+f(t) \lim _{h \longrightarrow 0} \frac{g\left(t+h e^{(\alpha-1) t}\right)-g(t)}{h} \\
& =\left(D^{\alpha} f\right)(t) \lim _{h \longrightarrow 0} g\left(t+h e^{(\alpha-1) t}\right)+f(t)\left(D^{\alpha} g\right)(t) .
\end{aligned}
$$

Since $g$ is continuous at $t$ then $\lim _{h \longrightarrow 0} g(t+$ $\left.h e^{(\alpha-1) t}\right)=g(t)$. This completes the proof of part $(4)$.

(5) can be proved in a similar way.
To prove (6), let $\varepsilon=h e^{(\alpha-1) t}$ in Definition 1. and then $h=\varepsilon e^{(1-\alpha) t}$. Therefore, 


$$
\begin{aligned}
\left(D^{\alpha} f\right)(t) & =\lim _{h \longrightarrow 0} \frac{f\left(t+h e^{(\alpha-1) t}\right)-f(t)}{h}\left(D^{\alpha} f\right)(t) \\
& =\lim _{\varepsilon \longrightarrow 0} \frac{f(t+\varepsilon)-f(t)}{\varepsilon e^{(1-\alpha) t}}\left(D^{\alpha} f\right)(t) \\
& =e^{(\alpha-1) t} \lim _{\varepsilon \longrightarrow 0} \frac{f(t+\varepsilon)-f(t)}{\varepsilon}\left(D^{\alpha} f\right)(t) \\
& =e^{(\alpha-1) t} f^{\prime}(t) .
\end{aligned}
$$

Theorem 3. Let b, c, $p \in \mathbb{R}$ and $0<\alpha \leq 1$. Then we have the following results:
(1) $\left.D^{\alpha}\left(t^{p}\right)=p e^{(\alpha-1) t}\right) t^{p-1}$ for all $p \in \mathbb{R}$.
(2) $D^{\alpha}(1)=0$.
(3) $D^{\alpha}\left(e^{c x}\right)=c e^{(\alpha-1) x} e^{c x}$, for all $c \in \mathbb{R}$.
(4) $D^{\alpha}(\sin (b x))=b e^{(\alpha-1) x} \cos (b x), b \in \mathbb{R}$.
(5) $D^{\alpha}(\cos (b x))=-b e^{(\alpha-1) x} \sin (b x), b \in \mathbb{R}$.

Theorem 4. However, it is worth noting the following conformable fractional derivatives of certain functions:

(1) $D^{\alpha}\left(\sin \left(1 / 1-\alpha e^{(1-\alpha) t}\right)\right)=\cos \left(1 / 1-\alpha e^{(1-\alpha) t}\right)$.

(2) $\left.\left.D^{\alpha}\left(\cos \left(1 / 1-\alpha e^{(1-\alpha) t}\right)\right)\right)=-\sin \left(1 / 1-\alpha e^{(1-\alpha) t}\right)\right)$.

(3) $D^{\alpha}\left(e^{1 / 1-\alpha e^{(1-\alpha) t}}\right)=e^{1 / 1-\alpha e^{(1-\alpha) t}}$.

(4) $D^{\alpha}\left(1 / 1-\alpha e^{(1-\alpha) t}\right)=1$.

Remark 2. In the case of the derivative proposed in [5], we have the following remark: a function could be $\alpha$ differentiable at a point but not differentiable, for example, take $f(t)=2 \sqrt{t}$, then $T_{1 / 2}(f)(0)=\lim _{t \longrightarrow 0^{+}} T_{1 / 2}(f)(t)=1$ where $T_{1 / 2}(f)(0)=1$ for $t>0$. But $T_{1}(f)(0)$ does not exist. This is not the case for the known classical fractional derivatives.

But for our definition, we have the same results of the classic case, and that is one more advantage of our derivative.

Next, we consider the possibility of $\alpha \in(n, n+1]$, for some $n \in \mathbb{N}$. We have the following definition.

Definition 2. Let $\alpha \in(n, n+1]$, for some $n \in \mathbb{N}$, and $f$ function be an $n$ differentiable at $t>0$. Then the $\alpha$ fractional derivative of $f$ is defined by

$$
\left(D^{\alpha} f\right)(t)=\lim _{h \longrightarrow 0} \frac{f^{(n)}\left(t+h e^{(\alpha-n-1) t}\right)-f^{(n)}(t)}{h},
$$

if the limit exists.
Remark 3. As a direct consequence of Definition 2, we can show that

$$
\left(D^{\alpha} f\right)(t)=t^{\alpha-n} f^{(n+1)}(t),
$$

where $\alpha \in(n, n+1]$ and $f$ is $(n+1)$ differentiable at $t>0$.

Remark 4. The previous definitions of fractional derivative Riemann-Liouville and Caputo do not enable us to study the analysis of $\alpha$ differentiable functions. However, our definition makes it possible to prove basic analysis theorems such as Rolle's theorem and the mean value theorem.

Theorem 5. Rolle's theorem for conformable fractional differentiable functions.

Let $a>0$ and $f:[a, b] \longrightarrow \mathbb{R}$ be a given function that satisfies

(1) $f$ is continuous on $[a, b]$,

(2) $f$ is $\alpha$-differentiable for some $\alpha \in] 0,1[$,

(3) $f(a)=f(b)$.

Then, there exists $c \in] a, b\left[\right.$, such that $f^{(\alpha)}(c)=0$.

Proof. Since $f$ is continuous on $[a, b]$, and $f(a)=f(b)$, there is $c \in] a, b$ [ which is a point of local extrema. With no loss of generality, assume $c$ is a point of local minimum. So

$$
\begin{aligned}
D^{\alpha} f(c) & =\lim _{\varepsilon \longrightarrow 0^{+}} \frac{f\left(c+\varepsilon c^{1-\alpha}\right)-f(c)}{\varepsilon} \\
& =\lim _{\varepsilon \longrightarrow 0^{-}} \frac{f\left(c+\varepsilon c^{1-\alpha}\right)-f(c)}{\varepsilon} .
\end{aligned}
$$

But, the first limit is nonnegative, and the second limit is nonpositive.

Hence $f^{(\alpha)}(c)=0$.

Theorem 6. Mean value theorem for conformable fractional differentiable functions).

Let (HTML translation failed) and $f:[a, b] \longrightarrow \mathbb{R}$, be $a$ given function that satisfies

(1) $f$ is continuous on $[a, b]$,

(2) $f$ is $\alpha$-differentiable for some $\alpha \in] 0,1[$.

Then, there exists $c \in] a, b[$ such that

$$
f^{(\alpha)}(c)=\frac{f(b)-f(a)}{(1 / \alpha-1) e^{(\alpha-1) b}-(1 / \alpha-1) e^{(\alpha-1) a}} .
$$

Proof. Consider the function

$$
g(x)=f(x)-f(a)-\frac{f(b)-f(a)}{(1 / 1-\alpha) e^{(1-\alpha) b}-(1 / 1-\alpha) e^{(1-\alpha) a}}\left(\frac{1}{1-\alpha} e^{(1-\alpha) x}-\frac{1}{1-\alpha} e^{(1-\alpha) a}\right) .
$$


Then,

$$
g(a)=f(a)-f(a)-\frac{f(b)-f(a)}{(1 / 1-\alpha) e^{(1-\alpha) b}-(1 / 1-\alpha) e^{(1-\alpha) a}}\left(\frac{1}{1-\alpha} e^{(1-\alpha) a}-\frac{1}{1-\alpha} e^{(1-\alpha) a}\right)=0
$$

and

$$
g(b)=f(b)-f(a)-\frac{f(b)-f(a)}{(1 / 1-\alpha) e^{(1-\alpha) b}-(1 / 1-\alpha) e^{(1-\alpha) a}}\left(\frac{1}{1-\alpha} e^{(1-\alpha) b}-\frac{1}{1-\alpha} e^{(1-\alpha) a}\right)=0
$$

Thus $g(a)=g(b)$. By Rolle's theorem, there exists $c \in] a, b\left[\right.$, such that $g^{(\alpha)}(c)=0$. Using the fact that $D^{\alpha}\left(1 / 1-\alpha e^{(1-\alpha) t}\right)=1$, the result follows.

Along the same lines in basic analysis, one can use the present mean value theorem to prove the following proposition.

Proposition 1. Let $f:[a, b] \longrightarrow \mathbb{R}$ be $\alpha$ differentiable for some $\alpha \in] 0,1[$.

(i) If $f^{(\alpha)}$ is bounded on $[a, b]$, where $a>0$, then $f$ is uniformly continuous on $[a, b]$, and hence, $f$ is bounded.

(ii) If $f^{(\alpha)}$ is bounded on $[a, b]$ and continuous at $a$, then $f$ is uniformly continuous on $[a, b]$, and hence, $f$ is bounded.

2.1. Fractional Integral. As in the work [5], it is interesting to note that, in spite of the variation of the definitions of the fractional derivatives, we can still adopt the same definition of the fractional integral here due to the fact that we obtained similar results in Theorem 4 as of the results (1)-(6) and (i)-(iii) in [5]. So, we have the following definition.

Definition 3. Let $\alpha \in(0,1)$ and $a \geq 0$, let $f$ be a function defined on $(a, t]$, Then, the $\alpha$ fractional integral of $f$ is defined by

$$
I_{\alpha}^{a}(f)(t)=\int_{a}^{t} e^{(1-\alpha) s} f(s) d s
$$

Theorem 7. If $f:[a, \infty) \longrightarrow \mathbb{R}$ is any continuous function in the domain of $I_{\alpha}$ and $0<\alpha \leq 1$. Then, for $t>a$, we have

$$
D_{a}^{\alpha} I_{\alpha}^{a} f(t)=f(t)
$$

Proof. Since $f$ is continuous, then $I_{\alpha}^{a} f(t)$ is clearly differentiable. Hence,

$$
\begin{aligned}
& D_{a}^{\alpha} I_{\alpha}^{a} f(t) \\
& \quad=e^{(\alpha-1) t} \frac{d}{\mathrm{~d} t} I_{\alpha}^{a}(f)(t) . \\
& \quad=e^{(\alpha-1) t} \frac{d}{\mathrm{~d} t} \int_{a}^{t} e^{(1-\alpha) s} f(s) \mathrm{d} s . \\
& \quad=e^{(\alpha-1) t} e^{(1-\alpha) t} f(t) . \\
& \quad=f(t) .
\end{aligned}
$$

Lemma 1. Let $f:(a, b) \longrightarrow \mathbb{R}$ be the function differentiable and $0<\alpha<1$. Then, for $t>a$, we have

$$
I_{\alpha}^{a} D_{a}^{\alpha} f(t)=f(t)-f(a)
$$

2.2. Applications. The authors of [5] introduced a new definition of fractional derivative to facilitate the calculations performed to solve the differential equation proposed by Professor S. Momani

$$
\left\{\begin{array}{l}
y^{(1 / 2)}+y=x^{2}+\frac{2}{\Gamma(2.5)} x^{3 / 2} \\
y(0)=0
\end{array}\right.
$$

but using the following approximation $\Gamma(2.5)=1.33$ to 1 .

Here we consider the following equation:

$$
\left\{\begin{array}{l}
y^{(1 / 2)}+y=x^{2}+2 x e^{-x / 2} \\
y(0)=0
\end{array}\right.
$$

Let us find a solution $y_{h}$ of the homogeneous equation $y^{(1 / 2)}+y=0$. We look for a solution of the form $y_{h}=e^{1-2 e^{x / 2}}$. It is easy to verify that $y_{p}(x)=x^{2}$ is a particular solution of the nonhomogeneous equation.

Now, the general solution is $y(x)=y_{h}(x)+y_{p}(x)=c e^{1-2 e^{x / 2}}+x^{2}$ where $c$ is a constant. Finally, the initial condition $y(0)=0$ implies that $c=0$. Hence $y(x)=x^{2}$.

Example 1. Based on the point (3) of Theorem 4, one can easily see that the auxiliary equation for $y^{(\alpha)}+y=0$ is 
$(\alpha-1) r+1=0$, so that the solution is given by $y(x)=e^{(1 / 1-\alpha) e^{(\alpha-1) x}}$.

The details of the solution of certain differential equations will be given later.

Remark 5. In the following example, we will show the benefit of the fractional derivative product rule which allows us to use the idea of the integrating factor

Example 2. Consider the following example:

$$
y^{(1 / 2)}+e^{-x / 2} y=x e^{-x} \text {. }
$$

By multiplying it by $e^{x}$, we obtain $e^{x} y^{(1 / 2)}+e^{x / 2} y=x$ and take advantage of the product rule for this fractional derivative (which is not possible with Riemann-Liouville and Caputo fractional derivatives): $\left(e^{x} y\right)^{(1 / 2)}=x$.

By using the fractional integral (18), we have $e^{x} y=\int_{0}^{x} t e^{t / 2} \mathrm{~d} t+C$.

Therefore, $y(x)=(2 x-4) e^{-x / 2}+c e^{-x}$.

Example 3.

$$
y^{(1 / 2)}=\frac{-y^{2}-x}{y e^{x / 2}} .
$$

Let us look for a differentiable solution $y$ which verifies this equation.

Since $D^{\alpha} y(x)=e^{(1-\alpha) x} y^{\prime}(x), \quad$ then $D^{1 / 2} y(x)=$ $y^{(1 / 2)}(x)=e^{-x / 2} y^{\prime}(x)$.

Thus, the fractional differential equation (25) becomes

$$
e^{-x / 2} y^{\prime}(x)=\frac{-y^{2}-x}{y} e^{-x / 2},
$$

which brings back to

$$
y^{\prime}+y=-\frac{x}{y}
$$

This is a differential equations of Bernouilli and can be solved easily.

Example 4. Consider now the fractional differential equation:

$$
y^{(1 / 2)}=\frac{y^{2}+x^{2} y+2 x^{4}}{x^{3} e^{x / 2}} .
$$

As before, the fractional differential equation (28) gives

$$
e^{-x / 2} y^{\prime}=\frac{y^{2}+x^{2} y+2 x^{4}}{x^{3} e^{x / 2}}
$$

From where

$$
x^{3} y^{\prime}+y^{2}+x^{2} y+2 x^{4}=0
$$

And this is a differential equation of Riccati.

\section{Data Availability}

No data were used to support this study.

\section{Conflicts of Interest}

The authors declare that there are no conflicts of interest regarding the publication of this paper.

\section{Authors' Contributions}

All the authors contributed equally to the writing of this paper. All the authors read and approved the final manuscript.

\section{References}

[1] K. S. Miller, An Introduction to Fractional Calculus and Fractional Differential Equations, J. Wiley and Sons, New York, NY, USA, 1993.

[2] A. Kilbas, H. Srivastava, and J. Trujillo, "Theory and applications of fractional differential equations," in Math. Studies, North-Holland, New York, NY, USA, 2006.

[3] K. Oldham and J. Spanier, The Fractional Calculus, Theory and Applications of Differentiation and Integration of Arbitrary Order, Academic Press, Cambridge, MA, USA, 1974.

[4] I. Podlubny, Fractional Differential Equations, Academic Press, Cambridge, MA, USA, 1999.

[5] R. Khalil, M. Al Horani, A. Yousef, and M. Sababheh, "A new definition of fractional derivative," Journal of Computational and Applied Mathematics, vol. 264, pp. 65-70, 2014.

[6] U. N. Katugampola, "A new fractional derivative with classical properties," e-print arXiv:1410.6535. 